\title{
MORE ON PHRAGMÉN-LINDELÖF FOR FUNCTION ALGEBRAS
}

\section{GLICKSBERG}

\section{Earlier Phragmén-Lindelöf-like results for a function algebra $A$ are extended to deal with possible unbounded behavior of an $A$-holomorphic function near a zero set rather than a peak set.}

The present note is intended to supplement an earlier one [2] in which Phragmén-Lindelöf arguments were applied to a uniform algebra $A$, with the usual behavior near $\infty$ replaced by behavior near a peak set. Here we shall exploit the fact that an arbitrary zero set can be converted to a peak set for a related algebra by slitting the spectrum along the inverse of an arc, imitating familiar techniques in the plane. We assume the reader is familiar with [2] and use much of the same notation.

Let $A$ be a uniform algebra with spectrum $M_{A}$ and let $X \subset M \subset M_{A}$ be two closed boundaries for $A$ (closed sets containing the Šilov boundary $\partial_{A}$ then). We shall say local maximum modulus holds (for $A$ ) on $M$ relative to $X$ (or simply, that $(M, X)$ satisfies l.m.m.) if each $m \in M \backslash X$ has a compact neighborhood $U \subset M \backslash X$ for which $|a| \leq \sup |a(\partial U)|$ on $U$, all $a \in A$. A fundamental observation about [2] that we shall need is that its results hold with $M_{A}$ and $\partial_{A}$ replaced by such a pair $M, X$. (There is one point in the proof of $\left[2\right.$, Th. 3] where $M$ seemingly must be $M_{A}$ : that $X$ there maps into $M$ (p. 404, 1.21). But in fact this follows trivially from the fact that $X$ is just the closure of $M$ in $M_{B_{0}}$.)

In all that follows we shall let $J_{0}$ be a simple closed arc joining 0 to $\infty$ in the Riemann sphere, with $J=J_{0} \backslash\{0\}$, and shall assume ${ }^{1}$

$$
\beta_{J}=\sup \left\{\arg w-\arg w^{\prime}: w, w^{\prime} \in J\right\}<\infty .
$$

$\beta_{J}$ plays the role of determining the allowable order of growth in our Phragmén-Lindelöf-like results. Note that our initial hypothesis on 1.m.m. is automatically satisfied if $M=M_{A}$ in all our results.

THEOREM 1. Suppose local maximum modulus holds on $M$ relative to $X, f \in A \backslash A^{-1}, g$ is continuous on $M \backslash f^{-1}(0), A$-holomorphic on $M \backslash\left(f^{-1}(0) \cup X\right)$, and, for some $k>0$ and $\beta, 0<\beta<\pi\left(2 \pi+\beta_{J}\right)^{-1}$, we have

$$
g \exp \left(\frac{-k}{|f|^{\beta}}\right) \quad \text { bounded on } \quad M \backslash f^{-1}(0)
$$


If $|g| \leq K$ on $\left(X \cup f^{-1}(J)\right) \backslash f^{-1}(0)$, then $|g| \leq K$ on $M \backslash f^{-1}(0)$.

Theorem 2. Suppose $(M, X)$ satisfies 1.m.m., $f \in A \backslash A^{-1}, g$ is bounded and continuous on $M \backslash f^{-1}(0), A$-holomorphic on $M \backslash\left(f^{-1}(0) \cup X\right)$, and

$$
c=\lim _{\substack{f(m) \rightarrow 0 \\ m \in f^{-1}(J) \cup X}} g(m)
$$

exists. Then $g$ has an extension in $C(M)$ constant on $f^{-1}(0)$, which, if $A$ is $X$-relatively maximal, lies in $A$.

Here " $X$-relatively maximal" means no properly larger subalgebra of $C(M)$ can have $X$ as a boundary. More generally, the boundedness of $g$ can be replaced by the hypothesis of Theorem 1 . Of more interest in applications is the following result.

Theorem 3. Suppose $(M, X)$ satisfies 1.m.m., $f \in A \backslash A^{-1}, g$ is continuous on $M \backslash f^{-1}(0)$, A-holomorphic on $M \backslash\left(f^{-1}(0) \cup X\right)$, bounded on $X \backslash f^{-1}(0)$, and satisfies (2). If

$$
|g(m)| \leq K|\log | f(m) \|, m \in f^{-1}(J)
$$

then $g$ is bounded and sup $\left|g\left(M \backslash f^{-1}(0)\right)\right|=\sup \left|g\left(X \backslash f^{-1}(0)\right)\right|$. Finally, if we have a finite limit

$$
c=\lim _{\substack{f(m) \rightarrow 0 \\ m \in X \cup f^{-1}(J)}} g(m)
$$

then $g$ has a continuous extension to $M, \equiv c$ on $f^{-1}(0)$, which lies in $A$ if $A$ is $X$-relatively maximal.

As an application we have ${ }^{2}$

Corollary 4. Suppose $f$ and $g$ are holomorphic on an open set $U$ in $\mathrm{C}^{n}$, and

$$
\left|\frac{g}{f}\right| \leq K|\log | f|| \quad \text { on } f^{-1}(J) .
$$

Then $g / f$ has an extension holomorphic on $U$.

Thus, in particular, boundedness along the one parameter family of varieties defined by $f$ and $J$ implies the boundedness of $g / f$. 
Our choice of the right side of (4) is somewhat arbitrary; the really relevant condition is simply that

$$
|g(m) \| f(m)|^{\varepsilon} \rightarrow 0 \text { as } \quad f(m) \rightarrow 0, m \text { in } f^{-1}(J)
$$

for every $\varepsilon>0$, as will be seen in the proof.

Let $f$ be as in Theorems $1-3$. Replacing $f, J$ by $e^{i \varphi} f, e^{i \varphi} J$ if necessary, we can assume that a branch of $\arg w$ can be defined on $\mathbf{C} \backslash J_{0}$ which varies between $\pi+\beta_{J} / 2$ and $-\pi-\beta_{J} / 2$ in $\mathbf{C} \backslash J_{0}$. We use this branch of arg to define powers $f^{\alpha}(0<\alpha<1)$ of $f$ on $M \backslash f^{-1}(J)$ which are $A$-holomorphic on $M \backslash f^{-1}\left(J_{0}\right)$, and we now let $M^{\wedge}$ be the spectrum of the closed selfadjoint subalgebra of $C\left(M \backslash f^{-1}(J)\right)$ generated by $A$ and the $f^{\alpha}$, so that each $a \in A$ (and each $f^{\alpha}$ ) has an extension $\hat{a}$ in $C\left(M^{\wedge}\right)$ while $M \backslash f^{-1}(J)$ is dense in $M^{\wedge}$. In fact the dual $\rho$ to $A \rightarrow C\left(M^{\wedge}\right)$ maps $M^{\wedge}$ into $M$ (because $\rho\left(M^{\wedge}\right)$ must lie in the closure of $M$ in $\left.M_{A}\right)$, and provides an inverse to the (trivially) continuous inclusion of $M \backslash f^{-1}(J)$ into $M^{\wedge}$. Thus $M \backslash f^{-1}(J)$ is imbedded homeomorphically in $M^{\wedge}$, and in fact $\rho$ is $1-1$ over $M \backslash f^{-1}(J)$ (so that $M \backslash f^{-1}\left(J_{0}\right)=\rho^{-1}\left(M \backslash f^{-1}\left(J_{0}\right)\right)$ is an open subset of $M^{\wedge}$ ): for if $\rho(x) \in M \backslash f^{-1}(J)$ then our branch of $z \rightarrow z^{\alpha}$ is continuous near $f(\rho(x))$, and so if $x_{\delta} \rightarrow x, x_{\delta} \in M_{\widehat{A}} \backslash f^{-1}(J)$, then $f\left(x_{\delta}\right)^{\alpha} \rightarrow f(\rho(x))^{\alpha}$, or $\hat{f^{\alpha}}\left(x_{\delta}\right) \rightarrow f^{\hat{\alpha}}(\rho(x))$, whence $f^{\hat{\alpha}}(x)=\hat{f}^{\alpha}(\rho(x))$, and, since $\hat{a}(x)=a(\rho(x))=$ $\hat{a}(\rho(x))$, no element of $C\left(M^{\wedge}\right)$ can distinguish $x$ and $\rho(x)$, and $x=\rho(x) \in$ $M \backslash f^{-1}(J)$.

In particular, $\rho$ is $1-1$ over $f^{-1}(0) \subset M \backslash f^{-1}(J)$; we can thus identify $\hat{f}^{-1}(0)$ and $f^{-1}(0)$, for if $x \in M^{\wedge}$ and $0=\hat{f}(x)$, then since $f \in A$, $f(\rho(x))=\hat{f}(x)$, so $\rho(x) \in f^{-1}(0)$ and thus $\rho(x)=x$. Since $\quad\left|\hat{f}^{\alpha}\right|=|\hat{f}|^{\alpha}$, $f^{\alpha-1}(0)=f^{-1}(0)$ as well.

Now let $B$ be the closed subalgebra of $C\left(M^{\wedge}\right)$ generated by $A^{\wedge}$ and the $\hat{f}^{\hat{a}}$. For $x \in M^{\wedge} \backslash \rho^{-1}\left(\hat{f}^{-1}\left(\mathrm{~J}_{0}\right) \cup X\right)=\rho^{-1}\left(M \backslash\left(f^{-1}\left(J_{0}\right) \cup X\right)\right)=$ $M \backslash\left(f^{-1}\left(J_{0}\right) \cup X\right)$ we have a compact neighborhood $U$ of $x$ in $M \backslash\left(f^{-1}\left(J_{0}\right) \cup X\right)$ on which all the $\hat{f}^{a}$ are uniformly approximable by elements of $A$ (because the power series for $z^{\alpha}$ about $\hat{f}(x)=f(\rho(x))$ has radius of convergence $|\hat{f}(x)|)$. Hence $|b(x)| \leq \sup |b(\partial U)|$ by local maximum modulus for $A$ on $M$ relative to $X$ and thus local maximum modulus holds for $B$ on $M^{\wedge}$ relative to $\rho^{-1}\left(f^{-1}\left(J_{0}\right) \cup X\right)$; in fact we see the least set forms a boundary for $B$. Because $\arg w$ varies between $\pi+\beta_{J} / 2$ and $-\pi-$ $\beta_{J} / 2$ on $\mathbf{C} \backslash J$, arg $f^{\alpha}(m)$ lies between $\pm \alpha\left(\pi+\beta_{J} / 2\right)$ for $m \in$ $M \backslash f^{-1}\left(J_{0}\right)$, and the same is thus true of arg $\hat{f}^{\alpha}$ on $M^{\wedge} \backslash f^{-1}(0)=$ $M^{\wedge} \backslash \hat{f}^{-1}(0)$, which lies in the closure of $M \backslash f^{-1}\left(J_{0}\right)=$ $\left(M \backslash f^{-1}(J)\right) \backslash f^{-1}(0)$ in $M^{\wedge}$. Consequently for $\alpha\left(\pi+\beta_{J} / 2\right)<1 / 2 \pi$ (or $\alpha$ 
$\left.<\pi\left(2 \pi+\beta_{J}\right)^{-1}\right), b=1-\hat{f^{\alpha}} / n$ peaks on $f^{-1}(0)=\left(\hat{f}^{\alpha}\right)^{-1}(0)$ for $n$ large; choosing $\alpha$ larger than our $\beta$ in Theorem 1, for $\gamma=\alpha^{-1} \beta<1$ we have $|1-b|^{\gamma}=1 / n \gamma\left|f^{\alpha}\right|^{\gamma}=1 / n \gamma|f|^{\alpha \gamma}=1 / n \gamma|f|^{\beta}$, and (2) says

$$
g \exp \left(\frac{-k}{n^{\gamma}|1-b|^{\gamma}}\right) \quad \text { is bounded on } M \backslash f^{-1}(0),
$$

in particular then on $M^{\wedge} \backslash \rho^{-1}\left(f^{-1}\left(J_{0}\right) \cup X\right)$. Consequently [2, Th. 1] applies to the continuous function $g{ }^{\circ} \rho$ on $M^{\wedge} \backslash f^{-1}(0)$ (which is $B$-holomorphic on $M^{\wedge} \backslash \rho^{-1}\left(f^{-1}\left(J_{0}\right) \cup X\right)$ and bounded on $\rho^{-1}\left(f^{-1}\left(J_{0}\right) \cup\right.$ $X)$ ) to assert that $g \circ \rho$ is bounded by $\sup \left|g \circ \rho\left(\rho^{-1}\left(f^{-1}(J) \cup X\right)\right)\right|=$ $\sup \left|g\left(f^{-1}(J) \cup X\right)\right|$, yielding the assertion of Theorem 1 .

To obtain Theorem 2 we have to first note that our $g, A$-holomorphic on $M \backslash\left(f^{-1}(0) \cup X\right)$, is $\mathbf{C}+f A$-holomorphic there: for $m$ in that set we have a compact neighborhood $U$ with $0 \notin b(f(U)$ ) (the closed convex hull) and for which, for $\varepsilon>0$, there is an $a \in A$ with $|g-a|<\varepsilon / 2$ on $U$. But $1 / f$ is uniformly approximable by polynomials in $f$ on $U$ since $0 \notin$ $\mathscr{b}(f(U))$, and thus we have $b \in A$ with $|(1 / f)-b|<\varepsilon / 2\|f\|\|a\|$ on $U$, so that $|g-a b f| \leq|g-a|+|a-a b f|<\varepsilon$ on $U$.

As a consequence we can replace $A$ by its closed subalgebra of all elements constant on $f^{-1}(0)$, and in effect reduce $f^{-1}(0)$ to a point; doing this first and constructing our algebra $B$ on $M^{\wedge}$ as before we have $f^{-1}(0)=$ $\hat{f}^{-1}(0)$ a peak point for $B$, and thus a zero set lying in the Choquet boundary. By (3), if we extend $g \circ \rho$ by giving it the value $c$ on $f^{-1}(0)$, its restriction to the boundary $\rho^{-1}\left(f^{-1}\left(J_{0}\right) \cup X\right)$ for $B$ is continuous, and the resulting function is $B$-holomorphic on $M^{\wedge} \backslash \rho^{-1}\left(f^{-1}\left(J_{0}\right) \cup X\right)$ and continuous on $M^{\wedge} \backslash f^{-1}(0)$; thus ${ }^{3}$ [2, Th. 3] applies to assert $g \circ \rho$, as extended, is continuous on $M^{\wedge}$, which of course means $g$, extended to be $c$ on $f^{-1}(0)$, is also continuous on $M$ with $f^{-1}(0)$ reduced to a point, hence on $M$, as asserted.

The final assertion follows from the extension [1, Th. 3.2] of Rado's theorem since $g$ is $A$-holomorphic on $M \backslash\left(X \cup g^{-1}(c)\right)$ (noting that $\partial_{A}$ can be replaced by a larger boundary there, with the interpretation of "relatively maximal" made in Theorem 2 ).

As mentioned, we can combine Theorems 1 and 2 to obtain

Corollary 5. Suppose $(M, X)$ satisfies l.m.m., $f \in A \backslash A^{-1}$, and $g$ is continuous on $M \backslash f^{-1}(0), A$-holomorphic on $M \backslash\left(f^{-1}(0) \cup X\right)$ and (2) and (3) hold. Then $g$ has an extension in $C(M)$ constant on $f^{-1}(0)$, which, if $A$ is $X$-relatively maximal, lies in $A$. 
Our proof of Theorem 3 depends on Theorem 2. We can assume $\|f\|$ $<1$, and will let $\|g\|_{X}=\sup \left|g\left(X \backslash f^{-1}(0)\right)\right|$. Because of (4) and the boundedness of $g$ on $X \backslash f^{-1}(0)$ we have that

$$
|f(m)|^{\varepsilon}|g(m)| \rightarrow 0 \quad \text { as } \quad|f(m)| \rightarrow 0, m \in f^{-1}(J) \cup X,
$$

and thus Corollary 5 applies to $f \in B \backslash B^{-1}, M^{\wedge}, \rho^{-1}\left(X \cup f^{-1}\left(J_{0}\right)\right)$ and the function $(g \circ \rho)$ for which (2) holds on $M^{\wedge} \backslash f^{-1}(0)$ while (3) holds because of (5); and of course it is $B$-holomorphic on $M^{\wedge} \backslash\left[f^{-1}(0) \cup\right.$ $\left.\rho^{-1}\left(f^{-1}\left(J_{0}\right) \cup X\right)\right]$. We conclude that

(6) $\hat{f}^{\hat{\varepsilon}}(g \circ \rho)$ is bounded by its bound on the $B$-boundary

$$
\rho^{-1}\left(f^{-1}\left(J_{0}\right) \cup X\right)
$$

(7) has a continuous extension to $M^{\wedge}$.

Because of (6) it suffices to know

$$
\left|f^{\varepsilon}\right||g| \leq\|f\|_{X}^{\varepsilon}\|g\|_{X} \quad \text { on } f^{-1}(J)
$$

for a sequence of $\varepsilon \rightarrow 0$. For then $\hat{f^{\varepsilon}}(g \circ \rho)$ is bounded by $\|f\|_{X}^{\varepsilon}\|g\|_{X}$ on $M^{\wedge}$, and letting $\varepsilon \rightarrow 0$ we get $|g(\rho(x))| \leq\|g\|_{X}$ for all $x$ in $M^{\wedge}$ with $\hat{f}(x) \neq 0$, i.e. for $x$ in $M^{\wedge} \backslash f^{-1}(0)$, which yields the first conclusion of Theorem 3; the second then follows from Theorem 2.

It remains then to show (8) holds for a sequence of $\varepsilon \rightarrow 0$. From the fact ((7) above) that $\hat{f^{\varepsilon}} \cdot(g \circ \rho)$ has a continuous extension to $M^{\wedge}, 0$ on $f^{-1}(0)$, we conclude that $|\hat{f}(g \circ \rho)|(x) \rightarrow 0$ as $\hat{f}(x) \rightarrow 0, x \in \hat{f}^{-1}\left(e^{i \varphi} \mathbf{R}_{+}\right), \mathbf{R}_{+}=(0$, $\infty)$, and thus that $|f(m)|^{\varepsilon}|g(m)| \rightarrow 0$ as $f(m) \rightarrow 0, m \in f^{-1}\left(e^{i \varphi} \mathbf{R}_{+}\right)$. Now replacing $J$ in our considerations by an arc such as $e^{i \varphi} \mathbf{R}_{+}$will change our $f^{\varepsilon}$ (and our $M^{\wedge}$ ) but does not of course change $\left|f^{\varepsilon}\right|=|f|^{\varepsilon}$; thus it suffices to know (8) with $J$ replaced by some one $\operatorname{arc} e^{i \varphi} \mathbf{R}_{+}$for $\varepsilon=\varepsilon_{j} \rightarrow 0$, and if (8) holds for one such arc it holds for all others, by Corollary 5 again, or, in effect by (6).

Now suppose (8) fails for all positive $\varepsilon \leq \varepsilon_{0}$. Then for each such $\varepsilon$ and $\operatorname{each} \varphi \in \mathbf{R}$,

$$
C_{\varepsilon, \varphi}=\sup \left\{|f(m)|^{\varepsilon}|g(m)|: f(m) \in e^{i \varphi} \mathbf{R}_{+}\right\}>\|f\|_{X}^{\varepsilon}\|g\|_{X},
$$

and in fact $C_{\varepsilon, \varphi}$ is independent of $\varphi$, since it is also $\sup \left\{|f(m)|^{\varepsilon}|g(m)|: m \in\right.$ $\left.M \backslash f^{-1}(0)\right\}$. 
We next observe that if $h=p \circ f$. where $p$ is a polynomial with $p(0)=$ 0 , then $|h(m)|^{\varepsilon}|g(m)| \rightarrow 0$ as $f(m) \rightarrow 0, f(m) \in e^{i \pi} \mathbf{R}_{+}$(since $|f(m)|^{e^{\prime}}|g(m)|$ $\rightarrow 0$ for all $\left.\varepsilon^{\prime}>0\right)$. In particular if we choose $p$ monotone on $(-1,0)$ so that $h^{-1}\left(e^{i \pi} \mathbf{R}_{+}\right)=f^{-1}\left(e^{i \pi} \mathbf{R}_{+}\right)$then

$$
|h(m)|^{\varepsilon}|g(m)| \rightarrow 0 \text { as } h(m) \rightarrow 0, m \in h^{-1}\left(e^{i \pi} \mathbf{R}_{+}\right) ;
$$

this is the case if $p(x)=x^{2} / 2+x$ for example, since $x^{2} / 2+x=-r<0$ and $-1<x<0$ iff $x=-1+\sqrt{1-2 r}$ and $0<r<1 / 2$, so that $(-1,0)$ $\cap p^{-1}(-\infty, 0)=(-1,0)$, and thus $h^{-1}(-\infty, 0)=f^{-1} p^{-1}(-\infty, 0)=$ $f^{-1}(-1,0)=f^{-1}(-\infty, 0)$.

So let $p(x)=x+x^{2} / 2, h=p \circ f$. Since $p^{-1}(0)$ meets the unit disc in $\mathbf{C}$ at precisely 0 (and $\|f\|<1), h^{-1}(0)=f^{-1}(0)$ as well, and thus if $(8)$ holds for $h$ in place of $f$ for $\varepsilon=\varepsilon_{j} \rightarrow 0$ we again could conclude that $g$ is bounded on $M \backslash h^{-1}(0)=M \backslash f^{-1}(0)$, which yields Theorem 3 as before. So now for $h$ we must have, as in (9)

$$
C_{\varepsilon}^{\prime}=C_{\varepsilon, \varphi}^{\prime}=\sup \left\{|h(m)|^{\varepsilon}|g(m)|: h(m) \in e^{i \varphi} \mathbf{R}_{+}\right\}>\|h\|_{X}^{\varepsilon}\|g\|_{X}
$$
where $C_{\varepsilon, \varphi}^{\prime}=C_{\varepsilon}^{\prime}$ is independent of $\varphi$ for all positive $\varepsilon \leq \varepsilon_{1}$. But in fact $C_{\varepsilon, \varphi}=$ $C_{\varepsilon}$ for $\varepsilon \leq \varepsilon_{0}$ in (9) implies $C_{\varepsilon, \pi}^{\prime} \neq C_{\varepsilon, 0}^{\prime}$ for $\varepsilon \leq \min \left(\varepsilon_{0}, \varepsilon_{1}\right)$ : for $p(x)=x+x^{2} / 2$ has the property that $|p(x)|>|x|$ for $x>0,|p(x)|<|x|$ for $-2 \leq x<0$, and thus, since the suprema in (9) and (10) must be assumed for $f(m)$ and $h(m)$ in $e^{i \varphi}\left(\delta_{\varepsilon}, 2\right)$, for $\delta_{\varepsilon}>0$, we have

$$
C_{\varepsilon, \pi}^{\prime}<C_{\varepsilon, \pi}=C_{\varepsilon, 0}<C_{\varepsilon, 0}^{\prime}
$$

so that (10) must fail, and (8) holds for $h$ in place of $f$ for some $\varepsilon_{j} \rightarrow 0$ completing our proof of Theorem 3.

One case in which (2) holds automatically is that dealing with the ratio of elements $f, g$ of $A$ : that $|g / f| \leq c \exp \left(1 /|f|^{\varepsilon}\right)$ is a trivial consequence of the boundedness of $t^{1 / \varepsilon} e^{-t}$ for $t>0$. Thus from Theorem 3 we obtain

Corollary 6. Suppose $(M, X)$ satisfies l.m.m., $f \in A \backslash A^{-1}, g$ is continuous on $M \backslash f^{-1}(0)$ and $A$-holomorphic on $M \backslash\left(f^{-1}(0) \cup X\right)$, while $g / f$ is bounded on $X \backslash f^{-1}(0)$. Then

$$
\left|\frac{g}{f}\right| \leq K|\log | f \| \text { on } f^{-1}(J)
$$

implies $|g / f|$ is bounded by its bound over $X \backslash f^{-1}(0)$. Moreover, if instead of (11) we have a finite limit 


$$
c=\lim _{\substack{f(m) \rightarrow 0 \\ m \in X \cup f^{-1}(J)}} \frac{g(m)}{f(m)}
$$

then $g / f$ has an extension in $C(M), \equiv c$ on $f^{-1}(0)$, which lies in $A$ if $A$ is $X$-relatively maximal.

Corollary 4 is one consequence. Indeed suppose $f$, $g$ are holomorphic on an open set $U \subset \mathbf{C}^{n},|g / f| \leq K|\log | f \|$ on $f^{-1}(J)$. For $z^{0} \in f^{-1}(0)$ we choose coordinates so that on a neighborhood $V$ of $z^{0}, f=p q$, where $q$ doesn't vanish on $V$ and $p$ is a Weierstrass polynomial, regular in $z_{n}$, say. Then as usual for $\varepsilon>0$ we have an $\delta>0$ for which $\left|z_{j}-z_{j}^{0}\right| \leq \delta, j \leq n-$ 1 , imply $p\left(z_{1}, \ldots, z_{n-1}, z\right)=0$ only if $|z|<\varepsilon / 2$, where we can assume the polycylinder $\Delta=\left\{z:\left|z_{j}-z_{j}^{0}\right| \leq \delta, j \leq n-1,\left|z_{n}-z_{n}^{0}\right| \leq \varepsilon\right\}$ lies in $V$. We now let $A=P(\Delta)$, the uniform algebra of functions continuous on $\Delta$ and analytic on $\Delta^{0}$, so that $M_{A}=\Delta$, and $\partial_{A}$ is the torus $\left\{z:\left|z_{j}-z_{j}^{0}\right|=\delta, j \leq n\right.$ $\left.-1,\left|z_{n}-z_{n}^{0}\right|=\varepsilon\right\}$, which we take as our $X$. Since local maximum modulus holds for $\left(M_{A}, \partial_{A}\right)$, and $\partial_{A} \cap f^{-1}(0)=\phi$ so we have $g / f$ bounded on $\partial_{A}$, Corollary 6 applies to show $g / f$ is bounded on $\Delta$, and so has a unique holomorphic extension to $\Delta^{0}$; thus $g / f$ has an extension holomorphic on $U$.

In the context of Corollary 4 it is rather obvious (from the fact that $f$ is an open mapping) that our bound over the set $f^{-1}(J)$ implies that $f^{-1}(0)$ $\subset g^{-1}(0)$, which the conclusion obviously also implies. (And of course in Corollary 6 it also follows that $f^{-1}(0) \subset g^{-1}(0)$, but this does not seem quite so obvious.)

Corollary 7. Suppose ( $M, X)$ satisfies 1.m.m., $f \in A \backslash A^{-1}$, and $F=$ $f^{-1}(0) \cap\left[X \cup f^{-1}(J)\right]^{-}$. Then $g \in A, g(F)=0$ imply $g\left(f^{-1}(0)\right)=0$.

For $g(F)=0$ says $\left(4^{\prime}\right)$ holds for $g$, so that, by Theorem 3, $g \mid\left(M \backslash f^{-1}(0)\right)$ has an extension in $C(M), 0$ on $f^{-1}(0)$, and thus $g\left(\partial f^{-1}(0)\right)$ $=0$. But $g=0$ on $f^{-1}(0) \cap X \subset F$ as well, and since $\partial f^{-1}(0) \cup\left(f^{-1}(0) \cap\right.$ $X)$ is a boundary for $A \mid f^{-1}(0)$ by local maximum modulus, $g\left(f^{-1}(0)\right)=0$.

Along somewhat similar lines, we note the following extension of the basic lemma of [1], which follows from it and our construction of $M^{\wedge}$.

Theorem 8. Suppose $(M, X)$ satisfies l.m.m. for $A$ and $f \in A$. For each neighborhood $V$ of $f^{-1}(0)$ in $X \cup f^{-1}\left(J_{0}\right)$ there is a neighborhood $U$ of $f^{-1}(0)$ in $M$ for which $g \in A$ and $g=0$ on Vimply $g=0$ on $U$.

Constructing our algebra $B$ on $M^{\wedge}$, there is a fixed neighborhood $V^{\wedge}$ 
of our peak set $f^{-1}(0)=\hat{f}^{-1}(0)$ in the boundary $\rho^{-1}\left(f^{-1}\left(J_{0}\right) \cup X\right)$ on which $\hat{g}$ vanishes for each such $g \in A$, whence by $[1,2.1]$ there is a neighborhood $W$ of $\hat{f}^{-1}(0)$ in $M^{\wedge}$ on which each $\hat{g}$ vanishes. But since $\rho\left(M^{\wedge} \backslash W^{0}\right)$ is a compact set disjoint from $f^{-1}(0)$ in $M$ we have a neighborhood $U$ of $f^{-1}(0)$ in $M$ with $U \cap \rho\left(M^{\wedge} \backslash W^{0}\right)=\phi$, and since

$$
\rho\left(M^{\wedge}\right)=\rho\left(M^{\wedge} \backslash W^{0}\right) \cup \rho\left(W^{0}\right)
$$

and

$$
M \subset \rho\left(M^{\wedge}\right) \cup f^{-1}\left(J_{0}\right), \quad U \subset \rho\left(W^{0}\right) \cup f^{-1}\left(J_{0}\right) .
$$

Finally, since all our $g^{\prime}$ s vanish on a fixed neighborhood $V \cap f^{-1}\left(J_{0}\right)$ of $f^{-1}(0)$ in $f^{-1}\left(J_{0}\right)$ and $g\left(\rho\left(W^{0}\right)\right)=\hat{g}\left(W^{0}\right)=0$, we can shrink our $U$ to obtain a neighborhood of $f^{-1}(0)$ in $M$ for which $g(U)=0$ for all $g \in A$ satisfying $g(V)=0$.

In particular, Theorem 8 says certain sets in $M$ cannot be zero sets: if $I$ is a closed nondegenerate arc in $\mathbf{C}$ and $f^{-1}(I) \neq \phi$ and misses $\partial_{A}$, then $f^{-1}(I) \neq g^{-1}(0)$ for any $g \in A$. Any $g \in A$ vanishing on $f^{-1}(I)$ necessarily vanishes on an open neighborhood of that set by Theorem 8 , whence $g^{-1}(0)=f^{-1}(I)$ implies $f^{-1}(I)$ is open and closed, and so necessarily meets $\left.\partial_{A}.\right)$

Remarks (Added June, 1974)

1. We can improve this last observation and Theorem 8: Suppose (M, $X)$ satisfy l.m.m. for $A$. For a closed non-degenerate arc $I$ in $\mathbf{C}$ and $f, g \in A$ with $\phi \neq f^{-1}(I) \subset g^{-1}(0) \backslash \partial_{A}$ we have $g \equiv 0$ on $f^{-1}(W)$, where $W$ is the component of I in $\mathbf{C} \backslash f\left(\partial_{A} \backslash g^{-1}(0)^{0}\right)$.

For convenience take $0 \in I, f^{-1}(0) \neq \phi$. We have $g=0$ near $f^{-1}(I)$ in $M$ by Theorem 8 (applied to subarcs), and so we can form the closed subalgebra $B$ of $C\left(M \backslash g^{-1}(0)^{0}\right)$ generated by $A$ and $1 / f$. Moreover since the elements of $B$ are $A$-holomorphic, by local maximum modulus we know

$$
\partial_{B} \subset\left(\partial_{A} \backslash g^{-1}(0)^{0}\right) \cup \partial g^{-1}(0)
$$

as in [1]. Because any point of $\partial_{B} \backslash\left(\partial_{A} \backslash g^{-1}(0)^{0}\right)$ would thus lie in the boundary of $g^{-1}(0)$ in $M \backslash g^{-1}(0)^{0}$, which is impossible by $[1,2.2]$, we conclude that $\partial_{B} \subset \partial_{A} \backslash g^{-1}(0)^{0}$.

Now if $W \cap f\left(M \backslash g^{-1}(0)^{0}\right) \neq \phi$ (so $W$ meets the spectrum $\operatorname{sp}_{B} f$ of $f$ relative to $B$ ) then since $0 \notin \mathrm{sp}_{B} f, W$ must meet the boundary of $\mathrm{sp}_{B} f$ 
which lies in $f\left(\partial_{B}\right)$. Since this implies $W \cap f\left(\partial_{A} \backslash g^{-1}(0)^{0}\right) \neq \phi$ which contradicts the definition of $W$, we conclude that $W \cap f\left(M \backslash g^{-1}(0)^{0}\right)=\varnothing$ so that $f^{-1}(W) \subset g^{-1}(0)^{0}$, yielding our assertion.

Note that the same argument applies if we suppose instead that $\phi \neq$ $f^{-1}(0) \subset g^{-1}(0)^{0} \backslash \partial_{A}$; moreover the role of $g^{-1}(0)$ can equally well be assumed by $\left\{m \in M: \lim a_{n}(m)=0\right\}$ where $\left\{a_{n}\right\}$ is a bounded sequence in $A$, if one uses $[1,2.5]$ in place of $[1,2.1]$ to provide the analogue of $[1,2.2]$.

Actually the fact that Theorem 8 provides a fixed neighborhood $U$ of $f^{-1}(I)$ in $M$ on which all $g=0$ on $f^{-1}(I)$ must vanish provides a further strengthening in case $M=M_{A}$, by a very similar argument.

THEOREM 8'. Suppose $K$ is nondegenerate compact connected set in $\mathbf{C}$, $f \in A$, and $H=\hat{f}^{-1}(K)$ is a hull-kernal closed subset of $M_{A}$. Then $\hat{f}^{-1}(K)$ $=\hat{f}(H)$ contains any component $W$ of $\mathbf{C} \backslash f\left(\partial_{A}\right)$ which it meets.

Since $M_{A}$ is the spectrum of $A$ and $\partial \operatorname{sp} f \subset f\left(\partial_{A}\right)$, we know $W \subset$ $\hat{f}\left(M_{A} \backslash \partial_{A}\right)$. So if $W \backslash \hat{f} \hat{f}^{-1}(K)=W \backslash K \neq \phi$ then its boundary in $W$ is nondegenerate, and so has a nondegenerate component which, necessarily, is arcwise connected. The component lies in $\partial K$ of course, so $\partial K$ contains a closed nondegenerate arc $I, I \subset W$. By Theorem 8 (applied to subarcs) we have a neighborhood $U$ of $\hat{f}^{-1}(I)$ in $M_{A}$ for which $g \in A, \hat{g}=0$ on $\hat{f}^{-1}$ (I) imply $\hat{g}(U)=0$, and thus $U$ lies in the hull of the kernel of $H=\hat{f}^{-1}(K)$, i.e., in $H$.

But $\hat{f}^{-1}(I) \subset U$ implies $\hat{f}^{-1}(I+\varepsilon D) \subset U$ for all small $\varepsilon>0$, where $D$ is the closed unit disc in $\mathbf{C}$. Taking $\varepsilon$ so small that $I+\varepsilon D \subset W \subset$ $\hat{f}\left(M_{A} \backslash \partial_{A}\right)$, we have

$$
\hat{f} \hat{f}^{-1}(I+\varepsilon D)=I+\varepsilon D \subset \hat{f}(U) \subset \hat{f}_{(H)}=\hat{f} \hat{f}^{-1}(K)=K,
$$

despite the fact that $I \subset \partial K$, our contradiction, showing $W \subset \hat{f} \hat{f}^{-1}(K)=$ $\hat{f}(H)$.

Note that the result of course fails trivially if $K$ is degenerate. As a special case we have the fact that any connected hull kernel closed subset $H$ of $M_{A} \backslash \partial_{A}$ is not the complete inverse of its image $K$ under any element $\hat{f}$ of $A^{\wedge}$, unless $\hat{f}$ is constant on $H$. (The same applies to any continuous $A$-holomorphic function $h$ on $M_{A}$ in place of $\hat{f}$ since the algebra generated by $A$ and $h$ has the same Šilov boundary and spectrum $[3,14.9]$.)

There are some interesting special cases. For example, since any interpolation set $H$ is hull kernel closed (as the spectrum of the quotient algebra $A \mid H=C(H)$ ), as all its closed sets must also be, $f \varepsilon A$ and $\hat{f}(H) \cap$ 
$\hat{f}\left(M_{A} \backslash H\right)=\phi$ imply $\hat{f}(H) \backslash \hat{f}(\partial)$ is totally disconnected. Indeed if $K$ is a nondegenerate compact connected subset of a component of $\hat{f}(H) \backslash \hat{f}(\partial)$ then $f^{-1}(K)$ is hull kernel closed, while $\hat{f} \hat{f}^{-1}(K)$ misses $\hat{f}(\partial)$, and so contains at least one component of $\mathbf{C} \backslash \hat{f}(\partial)$, by Theorem $8^{\prime}$, which of course implies the compact set $K$ meets $\hat{f}(\partial)$, our contradiction. Thus in particular, no $\in$ $A$ can map an interpolation set $H$ into a nondegenerate continuum $K \subset \mathbf{C}$ and $M_{A} \backslash H$ into $\mathrm{C} \backslash K$ unless $K \subset f(\partial)$.

2. As we observe in footnote 3 , we only needed a very weak form of [ 2 , Th. 3] in proving our Theorem 2, and of course there are stronger results which more fully utilize that earlier result. For example,

Theorem 2'. Suppose $(M, X)$ satisfies l.m.m., $f \in A \backslash A^{-1}, g$ is bounded on $M$, continuous on $M \backslash f^{-1}(0)$, and $A$-holomorphic on $M \backslash\left(f^{-1}(0) \cup X\right)$, while $g \mid\left(f^{-1}\left(J_{0}\right) \cup X\right)$ is continuous and $f^{-1}(0)$ carries unique Jensen measures for points in $f^{-1}(0)$. Then $g \in C(M)$, and $g \in A$ if $A$ is $X$-relatively maximal.

Thus in effect we replace (3) by continuity of $g$ on $f^{-1}\left(J_{0}\right) \cup X$, provided $f^{-1}(0)$ is rather special. In this form we require a slight improvement of [2, Th. 3]; note [2, p. 405, next to last paragraph] that uniqueness of Jensen measures is an adequate replacement for the uniqueness used there. For the proof of $2^{\prime}$ one constructs $B$ as before but without reducing $f^{-1}(0)$ to a point. Then $f^{-1}(0)$ appears as a peak set for $B$, and any measure on $X$ representing a point of $f^{-1}(0)$ on $B$ must be carried by $f^{-1}(0)$; thus the points of $f^{-1}(0)$ have unique Jensen measures (since such measures are necessarily Jensen for $A$ ) and [2, Th. 3] applies to assert $g \in C(M)$. Finally, our hypothesis implies $f^{-1}(0) \backslash X$ is all boundary, since otherwise we have a nonvoid open $U$ therein, and by 1.m.m. for $m \in U$ we have a Jensen measure carried by $\partial U \subset f^{-1}(0) \backslash\{m\}$, contradicting our uniqueness hypothesis. Since $f^{-1}(0) \backslash X$ is all boundary the arguments of [1] show the subalgebra of $C(M)$ generated by $A$ and $g$ has $X$ as a boundary, whence $g \in A$ if $A$ is $X$-relatively maximal.

Of course our hypothesis on $f^{-1}(0)$ is quite strong, so it may be worth noting that the result applies whenever $g$ is continuous on a quotient of $f^{-1}\left(J_{0}\right) \cup X$ obtained by injecting it in the spectrum of a subalgebra $A_{0}$ of $A$, provided the image of $f^{-1}(0)$ carries unique Jensen measures for $A_{0}$. In particular, for $A_{0}$ the closure of $\mathbf{C}+f A$, Theorem 2 reappears.

3. Next we want to point out a consequence of our construction which 
shows the range of $f$ near certain subsets $F$ of $f^{-1}(0) \backslash X$ cannot be too small; for the special case of $M=M_{A}$ and $F=f^{-1}(0) \subset M \backslash X$ it follows directly from Theorem 8 and the Šilov idempotent theorem.

THEOREM 9. Suppose $(M, X)$ satisfies l.m.m., $f \in A \backslash A^{-1}, F$ in $f^{-1}(0) \backslash X$ is a peak set for the uniform closure $\left(A \mid f^{-1}(0)\right)^{-}$of $A \mid f^{-1}(0)$, while $F \cap \partial f^{-1}(0) \neq \phi$ if $M \neq M_{A}$. Then for any $\varepsilon>0$ and neighborhood $V$ of $F$ in $M \backslash X$, no curve joins 0 to $\{z:|z|=\varepsilon\}$ in $0 \cup(\mathbf{C} \backslash f(V))$.

In particular this applies to any peak point for $\left(A \mid f^{-1}(0)\right)^{-}$lying off $X$ (and consequently in $\partial f^{-1}(0)$ by l.m.m.). In fact if $M=M_{A}$ it applies to any component $F$ of $f^{-1}(0)$ which misses $X$, since any neighborhood $V$ of $F$ contains another, $W$, with $f^{-1}(0) \cap W$ open and closed in $f^{-1}(0)$, and so a peak set for $\left(A \mid f^{-1}(0)\right)^{-}$by Šilov's theorem (since $f^{-1}(0)$ in $M_{A}$ is the spectrum of this algebra). Thus our result applies to the neighborhood $V$ of $F_{0}=f^{-1}(0) \cap W$ to yield the conclusion.

Now suppose our result fails. Then we can extend our curve to a $J_{0}$ joining 0 to $\infty$, with $f(V) \cap\{z:|z|<\varepsilon\} \cap J_{0}=\{0\}$, and then slit $M$ and construct our algebra $B$ as before, making $f^{-1}(0)$ into a peak set for $B$. But recall that $\rho^{-1}\left(M \backslash f^{-1}(J)\right)=M \backslash f^{-1}(J)$ so that $\rho\left(M^{\wedge} \backslash M\right) \subset f^{-1}(J)$. As a consequence some neighborhood $W \subset V \cap f^{-1}\{z:|z|<\varepsilon\}$ of $F$ in $M$ remains a neighborhood in $M^{\wedge}$ : otherwise a net $\left\{m_{\delta}\right\}$ in $M^{\wedge} \backslash M$ converges to $x \in F \subset f^{-1}(0)$, whence $\rho\left(m_{\delta}\right) \rightarrow \rho(x)=x$ and $f\left(\rho\left(m_{\delta}\right)\right) \rightarrow f(x)$ $=0$, so that, since $\rho\left(m_{\delta}\right) \in f^{-1}(J), f\left(\rho\left(m_{\delta}\right)\right) \in f(V) \cap\{z:|z|<\varepsilon\} \cap J=$ $\phi$ for $\delta \geq \delta_{0}$, our contradiction. Evidently $W \cap X \subset V \cap X=\phi$.

Now $B \mid f^{-1}(0)$ is closed in $C\left(f^{-1}(0)\right)$, and so contains the closure $\left(A \mid f^{-1}(0)\right)^{-}$of $A \mid f^{-1}(0)$ in $C\left(f^{-1}(0)\right)$. On the other hand $B \mid f^{-1}(0) \subset$ $\left(A \mid f^{-1}(0)\right)^{-}$since polynomials in the various $\hat{f}^{\alpha}$ with coefficients in $\hat{A}$ are dense in $B$, and these restrict to elements of $\hat{A} \mid f^{-1}(0)$. Thus $B \mid f^{-1}(0)=\left(A \mid f^{-1}(0)\right)^{-}$, so that $F$ is a peak set within $f^{-1}(0)$ of $B$; since $f^{-1}(0)$ is a peak set for $B, F$ is itself a peak set for $B$ by Bishop's lemma. But in the boundary $\rho^{-1}\left[X \cup f^{-1}\left(J_{0}\right)\right]$ for $B, f$ vanishes on the neighborhood

$$
\begin{gathered}
\rho^{-1}\left[W \cap\left(X \cup f^{-1}\left(J_{0}\right)\right)\right]=\rho^{-1}\left[W \cap f^{-1}\left(J_{0}\right)\right] \\
=\rho^{-1}\left[W \cap f^{-1}(0)\right]=W \cap f^{-1}(0) \text { of } F,
\end{gathered}
$$

and thus $f$ vanishes near $F$ in $M^{\wedge}$, hence near $F$ in $M$, exactly as in the proof of Theorem 8 .

Now $F \subset f^{-1}(0)^{0}$ so we have clearly reached our final contradiction if 
$F \cap \partial f^{-1}(0) \neq \varnothing$, and it only remains to see this hypothesis is redundant if $M=M_{A}$. In that case we let $W$ be the neighborhood $f^{-1}(0)^{0} \backslash X$ of $F$ in $M$, and note that we have a uniform limit $h$ on $f^{-1}(0)$ of elements in $A$, for which $h(F)=1$ and $|h|<1$ on $f^{-1}(0) \backslash U$, where $U$ is a neighborhood of $F$ with $U^{-} \subset W$. Thus $\sup \left|h\left(W^{-} \backslash W\right)\right|<1$ so that for some approximating $a$ in $A$ and some $m \in F,|a(m)|=1$ and $\sup \left|a\left(W^{-} \backslash W\right)\right|<1$, contradicting Rossi's local maximum modulus theorem.

Evidently the distinction between our two cases arises from the fact that we have chosen a weaker notion of local maximum modulus for a pair $(M, X)$ than actually obtains for $\left(M_{A}, \partial_{A}\right)$, in using "some" neighborhood, rather than "all."

4. Finally we should note the applicability of Theorem 2 and Corollary 6 to some relatives of the question of which functions operate on $A$. Applied directly to that question the first yields only a very special case of the result of de Leeuw and Katznelson [3]; it just shows that if $A$ contains an element $a_{0}$ for which $a_{0}\left(M_{A}\right) \neq a_{0}(\partial)$ then any $\phi$ defined on an open subset $U$ of $\mathbf{C}$ for which $\dot{a} \in A$ implies $\phi(a) \in A$ must be analytic (while [3] shows this for a general nonself-adjoint uniform algebra). Indeed if $\phi$ is not analytic one has a nearby $C^{(1)}$ nonanalytic $\psi$ which operates (using convolution exactly as in [3]). Now for any $z$ in the domain of $\psi$ and $m_{0}$ with $a_{0}\left(m_{0}\right) \notin a_{0}(\partial)$ we can replace $a_{0}$ by $a=c a_{0}+d, c, d \in \mathbf{C}$, so as to get $a\left(M_{0}\right)=z \notin a(\partial)$, and since $\psi$ is $C^{(1)}$

$$
\lim _{\substack{a(m)-z \rightarrow 0 \\ a(m)-z \in R_{+}}} \frac{\psi(a(m))-\psi(z)}{a(m)-z}
$$

exists; since the ratio is $A$-holomorphic off $a^{-1}(z)=(a-z)^{-1}(0)$, the limit exists without the second restriction by Theorem 2 , and since a neighborhood of $z$ lies in the spectrum of $a$, that implies $\psi$ satisfies the Cauchy-Riemann equations at $z$, yielding our contradiction.

4.1. As a variant, suppose $\phi$ is $C^{(1)}$ on an open set $U \subset a\left(M_{A}\right) \backslash a(\partial)$, while for each $z \in U$ we have $\phi(a)$, on $a^{-1}\left(z+\left[-\varepsilon_{z}, \varepsilon_{z}\right]+i\left[-\varepsilon_{z}, \varepsilon_{z}\right]\right)$, a uniform limit of a bounded sequence in $A$ ( $\varepsilon_{z}>0$ of course). Then $\phi$ is analytic on $U$. This follows from the argument below for our next remark, but is easily seen when $\phi(a)$ coincides with an element $b=b_{z}$ of $A$ on $a^{-1}(z$ $\left.+\left[-\varepsilon_{z}, \varepsilon_{z}\right]+i\left[-\varepsilon_{z}, \varepsilon_{z}\right]\right)$ for each $z \in U$; taking $z=0$, if $a\left(m_{0}\right)=z=0$ we have 


$$
\frac{b-b\left(m_{0}\right)}{a-a\left(m_{0}\right)}=\frac{b-b\left(m_{0}\right)}{a}
$$

$A$-holomorphic off $a^{-1}(0)$, and bounded on $a^{-1}(\mathbf{R}+)$ where it has a limit as $a(m) \rightarrow 0$. Thus by the second half of Corollary 6 there is an unrestricted limit as $a(m) \rightarrow 0$, so since $U$ lies in the spectrum of $a$ this says

$$
\begin{aligned}
\lim _{h \rightarrow 0 \text { in } \boldsymbol{R}} \frac{\varphi(z+h)-\varphi(z)}{h} & =\lim _{a(m) \rightarrow 0 \text { in } \boldsymbol{R}} \frac{b(m)-b\left(m_{0}\right)}{a(m)} \\
& =\lim _{a(m) \rightarrow 0 \text { in } i \boldsymbol{R}} \frac{b(m)-b\left(m_{0}\right)}{a(m)} \\
& =\lim _{h \rightarrow 0 \text { in } \boldsymbol{R}} \frac{\varphi(z+i h)-\varphi(z)}{i h}
\end{aligned}
$$

so that $\phi$ satisfies the $C$. $-R$. equations at $z=0$.

The more general assertion follows from the same argument as our final remark where again $M=M_{A}$ : if $a^{-1}(0) \cap \partial=\varnothing$ then $a^{-1}([-\varepsilon, \varepsilon]+$ $i[-\varepsilon, \varepsilon])=F$ has the property that for any $\phi$ which is $C^{(1)}$ near 0 and does not satisfy the C.-R. equations at $0, \phi(a)$ is not the uniform limit on $F$ of a bounded sequence $\left\{a_{n}\right\}$ in $A$. (Since $P(a(F))=C(a(F)), \phi(a)$ is in the uniform closure $(A \mid F)^{-}$of $A \mid F$, and so $(A \mid F)^{-} \neq \tilde{A}_{F}$, the uniform limits on $F$ of bounded sequences in $A$.)

Indeed otherwise, by the argument of the latter half of $[1,2.5]\left\{a_{n}\right\}$ converges uniformly on a neighborhood $V$ of $a^{-1}(0)$ to some function $b$, and we can assume $V \cap \partial=\phi$, in fact, that $V=a^{-1}(r D)$, where $D$ is the closed unit disc and $r>0$ is small, and then $V$ is precisely the spectrum of $(A \mid V)^{-}$. So since $\partial V$ contains the Silov boundary of $(A \mid V)^{-}, b \in(A \mid V)^{-}$, $a^{-1}(0) \cap \partial V=\phi$ while $b=\phi(a)$, on $a^{-1}([-\varepsilon, \varepsilon]+i[-\varepsilon, \varepsilon]) \cap V=$ $(a \mid V)^{-1}([-\varepsilon, \varepsilon]+i[-\varepsilon, \varepsilon])$, we now have precisely, for $(A \mid V)^{-}$, the situation in the already treated special case of 4 . 1 , so the $C$. $-R$. equations for $\phi$ at 0 follows as there, our contradiction.

(In the same vein, if $\phi$ is $C^{(1)}$ and nowhere analytic $\left(\phi(z)=|z|^{2} \cdot z+\bar{z}\right.$ for example), $a \in A$ and $\phi(a) \in A$ then $a\left(M_{A}\right)=a(\partial)$ : otherwise for $m$ with $a(m) \notin a(\partial),(\phi(a)-\phi(a(m)) /(a-a(m))$ has a limit as $a \rightarrow a(m)$, so that $\phi$ satisfies the $C$. $-R$. equations at $a(m)$.) 


\section{FOOTNOTES}

${ }^{1}$ As will be seen, $J_{0}$ can always be replaced by a neighborhood of 0 in $J_{0}$ extended by part of a ray through the origin; thus $\beta_{J}$ really depends on $J$ near 0 . In fact (1) is not needed in Theorems 2 and 8.

${ }^{2}$ This much weaker than what can be obtained if one is willing to use more of the theory of several complex variables, as was pointed out to me by $\mathrm{H}$. Alexander: it suffices to assume our bound only on $f^{-1}\left(\left\{z_{n}\right\}\right)$, for some sequence $z_{n} \rightarrow 0$ in C. (For this implies $f^{-1}(0) \subset g^{-1}(0)$, and at each point of $f^{-1}(0)$ the unique factorization of germs and the nullstellensatz imply that $f$ must divide $g$ locally.)

${ }^{3}$ After this was written I noted that a much simpler argument applies here since we are dealing with a peak set. Indeed, just the classical argument $[5$, p. 179] with $z$ replaced by $1 / \hat{f}$ applies: for suppose $g$ is a bounded $A$-holomorphic function off $f^{-1}(0)$ which $\rightarrow 0$ as $f \rightarrow 0$ in $X$, where $f \in A$ has $|\arg f|<\pi / 4$, and $|g| \leq K$. Choose $\delta>0$ so $|g|<\varepsilon$ on $f^{-1}(\delta D) \cap X$ and set $G=g f^{-1} /\left(f^{-1}+\lambda\right)=g /(1+\lambda f) . \lambda>0$, so $|G| \leq|g| \leq K$ and on $f^{-1}(\delta D) \cap X,|G| \leq|g|<\varepsilon$. On $X \backslash f^{-1}(\delta D),|G| \leq K / \lambda|f|$, and so will be $<\varepsilon$ if we set $\lambda=K / \varepsilon \inf f\left(X \backslash f^{-1}(\delta D) \mid(\right.$ which is $\leq K / \varepsilon \delta)$. But now $|G|<\varepsilon$ on $X \backslash f^{-1}(0)$, and so on $M_{A} \backslash f^{-1}(0)$ by $[1,4.8]$. So $|g| \leq(1+$ $\lambda|f|)|G|<\varepsilon$ on $\{m: 1+\lambda|f(m)|<2\}$.

In fact this argument does not apply in the setting of [2, Th. 3], since a zero set in the Choquet boundary need not be a peak set; for example, if $A$ is Basener's algebra [4, p. 202] built over a compact $E \subset \mathrm{C}$ for which $R(E)$ is regular, and $F \subset E$ is a zero set for $R(E)$ which is not a peak set, then the points of $M_{A}$ over $F$ cannot form a peak set for $A$, but do form a zero set.

\section{REFERENCES}

1. I. Glicksberg, Maximal algebras and a theorem of Rado, Pacific J. Math., 14 (1964), 919-941; correction 19 (1966), 587.
2. (1967), 401-406.
A Phragmén-Lindelöf theorem for functional algebras, Pacific J. Math., 22

3. K. de Leeuw and Y. Katznelson, Functions that operate on non-self adjoint algebras, J. d'Analyse Math. 11 (1963), 207-219.

4. E. L. Stout, The Theory of Uniform Algebras, Bogden and Quigley, Tarrytown on Hudson, New York, 1971.

5. E. C. Titchmarsh, The Theory of Functions, Oxford University Press, 2nd Ed., 1937.

Received March 2, 1973. Work supported in part by the N. S. F.

UNIVERSITY OF WASHINGTON

AND

OXFORD UNIVERSITY 